\title{
LXII. Experimental determination of the ionization potential of helium
}

\section{Charles B. Bazzoni Ph.D.}

To cite this article: Charles B. Bazzoni Ph.D. (1916) LXII. Experimental determination of the ionization potential of helium , Philosophical Magazine Series 6, 32:192, 566-575, DOI: $10.1080 / 14786441608635605$

To link to this article: http://dx.doi.org/10.1080/14786441608635605

册 Published online: 08 Apr 2009.

Submit your article to this journal $\sqsubset x$

Џ Article views: 2

Q View related articles $\square$

Citing articles: 2 View citing articles $\square$ 
a rectangular hyperbola. Beyond this critical bend, the curve approaches more or less rapidly to a straight line parallel to the axis of abscisse. The above, in connexion with previous results on the collapsing pressures of long tubes, thus should allow us to calculate with reasonable approximation the collapsing pressures of short steel tubes of these thicknesses.

Laboratory of Physics, University of Illinois, Urbana, Illinois, May 1916.

LXII. Experimental Determination of the Ionization Potential of Helium. By Charles B. Bazzoni, Ph.D., Harrison Research Fellow, University of Pennsylvania*.

$\mathrm{r}$ 'HE energy of impact necessary to remove an electron 1 from simple atoms can be calculated directly from Bohr's theory. The application of Bohr's formula to helium indicates that an energy corresponding to that of an electron falling through $29 \cdot 3$ volts is necessary to remove one electron from the helium atom, and that an energy corresponding to a fall through 83.4 volts is necessary to remove both electrons. If the theory is correct, no assumed configuration of the electrons in the atom can give any other probable values but these, provided that impact ionization, so called, results directly and solely from impacts as is generally assumed. It follows that an experimental determination of the ionization potential of helium ought to serve as an excellent direct check on the validity of Bohr's theory itself. The first determination of this potential was made by Franck and Hertz $\dagger$ using a very sensitive method for detecting small amounts of impact ionization. The value obtained was $20 \cdot 5$ volts. The only other determination has been made by Pawlow $\ddagger$, who used a method essentially the same as Franck and Hertz, and got a result, 20 volts, in excellent agreement with those investigators. Pawlow, however, found it unnecessary to apply any correction for the initial velocity of the impinging electrons, while Franck and Hertz's result involves such a correction. Recently $\mathrm{K}$. Compton $\$$ has calculated the ionization potential, making use of a formula which he has developed and of various recorded data on heliam ionization. He obtains 22.5 volts

* Communicated by Prot. O. W. Richardson, F.R.S.

+ Ber. der D. Phys. Ges. 1913, p. 34.

$\ddagger$ Proc. Roy. Sac. vol. xc. p. 338 (1914).

\$ Phys. Rer. 2nd ser. vol. vii. pp. 4 \& 5. 
as the most probable value. All of these values are too low to fit into Bohr's theory. Bohr has suggested that the effects observed in the experiments might have been due to ionization of impurities by radiation from the helium under 20.5 volt bombardment, or to the liberation of electrons from the metal parts of the apparatus from the same cause. The fact that the characteristic frequency of helium as calculated by Cuthbertson * from dispersion data $\left(5.9 \times 10^{15}\right)$ corresponds very closely to 20.5 -volt impacts, lends some support to this explanation. Further, certain experiments of Rau's $\dagger$ in which he finds 30 volts necessary to excite the ordinary many-lined spectrum of helium and 80 volts necessary to excite $\lambda 4686$ have been taken, although without sufficient justification $\ddagger$, to imply that Bohr's calculated value is the correct one. In view of the remarkable agreement with fact of many of the deductions from this theory of Bohr's, it bas seemed worth while to re-determine the ionization potential of helium, avoiding the suggested possibilities of error, and, further, in view of the extraordinary susceptibility of helium to disturbances due to minute traces of impurities, with an arrangement such that all traces of occluded gases could positively be got rid of in the first place and the gas subsequently be maintained absolutely without contamination. The apparatus used in this investigation has been designed with these ends in view.

Fig. 1.

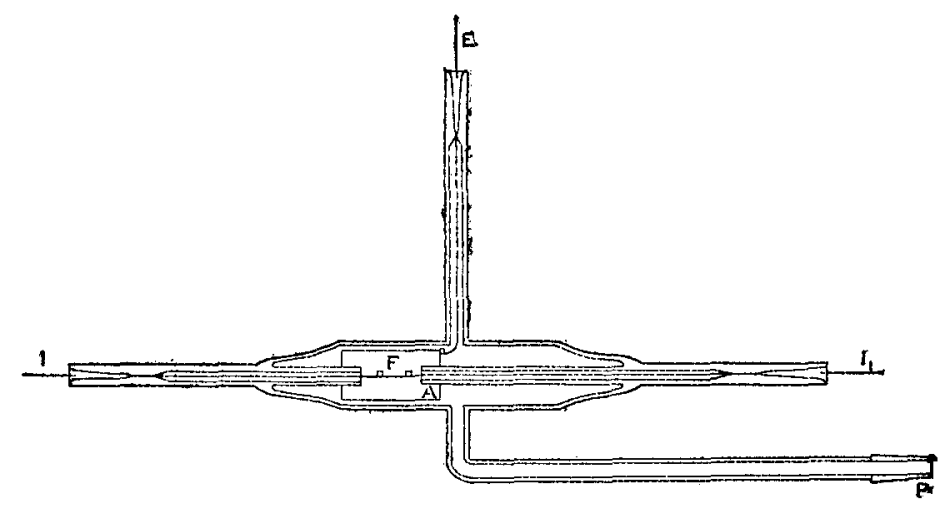

The experimental tube, which is constructed of transparent quartz, is shown in fig. 1. The filament $F$, which is of

* Proc. Roy. Soc. 1910.

† Sitz. der Phys. Med. Ges. zu Wïrzburg, Feb. 1914.

‡ Richardson \& Bazzoni, 'Nature,' Sept. $7,1915$. 
tungsten about $.8 \mathrm{~cm}$. in length and $.08 \mathrm{~cm}$. in diameter, was surrounded by a coaxial cylinder of sheet copper (A), the lead (E) from which was connected with a quadrant electrometer. As the currents dealt with were sometimes of considerable magnitude, the electrometer was provided with an adjustable capacity. The sensibility of the electrometer with no capacity was roughly $600 \mathrm{~mm}$. per volt. At $\mathrm{P}$ is a ground cone for connecting the tube with the air-pump and with the apparatus for producing and purifying the helium. The joint at this cone is preferably covered with sealing-wax without the use of tap-grease, but in these experiments it was actually covered with soft wax, which seemed a satisfactory arrangement. The heating current was taken from a 50-volt storage battery, and, since it was absolutely essential that the temperature of the filament should be maintained constant over relatively long periods, the filament was put in one arm of a Wheatstone bridge, which was kept accurately balanced by the use of three continuously adjustable resistances in parallel in the main circuit. I'his heating circuit was insulated from the ground. The negative end of $F$ was connected to a sliding contact on a rheostat, which was in series with a 200 -volt storage battery. The potentials were measured directly through a high-resistance Weston standard voltmeter between the negative end of $\mathrm{F}$ and the ground. This voltmeter was checked against a Clark cell and a standard resistance, and found correct within $0 \cdot 1$ volt over the range used.

Connected to $\mathrm{P}$ there was first a discharge-tube for examining the purity of the gas with a spectroscope; next a U tube to be surrounded with liquid air to get rid of mercury; next a mercury cut-off; then a $U$ tube with charcoal; then another mercury cut-off; then a dischargetube containing phosphorus pentoxide. There was next a stopcock beyond which was a quartz tube containing clevite, with a lateral tube containing potassium permanganate crystals and a final connexion with the pump. Since the ionization potential of helium is higher than that of any of the substances with which it may be expected to be contaminated, it is of the highest importance to get the gas absolutely pure. In this arrangement the major portion of the hydrogen was removed by splarking for a long time with oxygen over phosphorus pentoxide. The residual hydrogen and other impurities were then taken out by the liquid air and charcoal. The gas finally obtained in the experimental tube was of a high degree of purity, and in the work that follows showed no trace, spectroscopically, of contamination 
with anything excepting where it is specifically stated otherwise. As might be expected, the main difficulty was in getting the occluded gas out of the cylinder $\mathbf{A}$ and in properly ageing the filament. The occluded gas was finally eliminated by running the pump steadily while maintaining the entire apparatus (excepting the seals $I, I_{1}$, and $E$ ) at a bright red heat for two hours by the use of a blowpipe. Spurious effects due to the filament surface were got rid of by glowing the filament out at a very high temperature during several hours. The only impurity which appeared subsequent to this treatment was mercury, which could, of course, be kept down only by the constant use of liquid air.

In addition to the points mentioned above, there is a difference between this apparatus and the arrangements used by Franck and Hertz and by Pawlow which might have been of considerable importance. These authors made use of a device by which a large number of electrons were subjected in a space $A$ to an accelerating field, after which they passed into a retarding field in a space $B$ where impact ionization occurred. The amount of impact ionization was measured by the number of positive ions collected by an electrode bounding space $B$, all of the negative electrons being returned by the field into the space A. Such an arrangement is very sensitive for detecting the potentials at which ionization sets in. but it has the disadvantage that it affords no opportunity for an accurate comparison between the extra current due to impact ionization and the electron current which is the cause of it. For this reason the results of such experiments may always be open to the objection that the impact ionization results observed are possibly due either to the presence of small amounts of some more easily ionizable impurity, or to the occurrence of multiple impacts under low voltages, which, although giving rise to some ionization, may only form an insignificant part of the impact ionization in the same gas when higher roltages are available. The present arrangement, on the other hand, measures directly both the primary electron current and the extra current it gives rise to by impacts.

A series of observations made before the last traces of occluded gases had been expelled is of interest in showing the marked effect of minute quantities of impurities. In fig. 2 we have the steady deflexion of the electrometer after a 30-second charge plotted against the negative driving potential from $F$ to $A$. The heating current and the capacity across the quadrants of the electrometer are shown 
570 Dr. C. B. Bazzoni : Experimental Determination

beside each curve. In A we have a fresh charge of prre gas-ionization by collisions is seen to set in at, roughly, 20 volts. In $B$, which was run with the same gas after the filament had been glowed for about an hour, the setting in

Fig. 2.

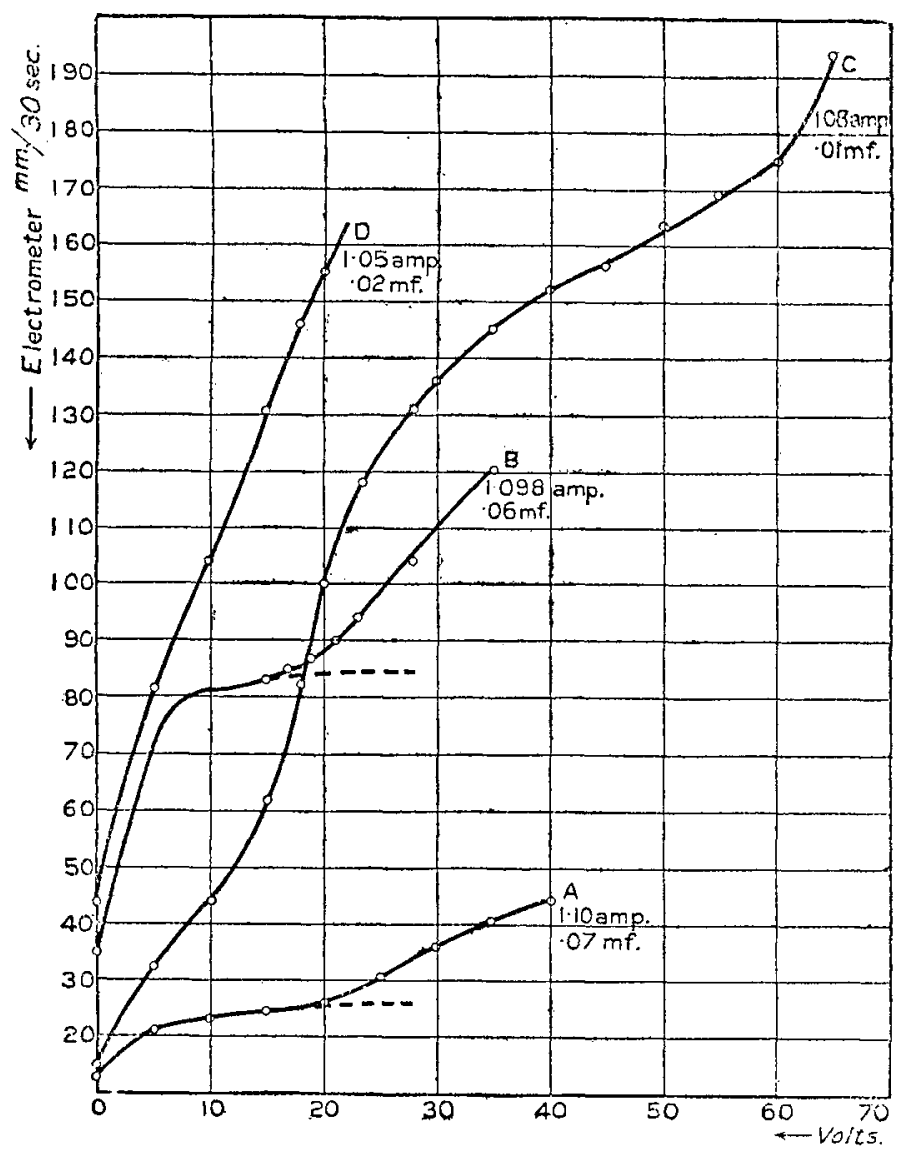

of ionization at 14 or 15 volts indicates the presence of hydrogen, although there was no certain spectroscopic evidence of this gas at this stage. In $\mathrm{C}$ we have the curve for the same gas when the spectroscope showed hydrogen faintly, and in $D$ the curve when hydrogen was plain in the spectrum together with a faint indication of $\mathrm{CO}$. In curve $\mathrm{C}$ there is evidence of the setting in of a second ionization above 
40 volts. This, together with the value of the primary ionization potential taken from $A$, is a preliminary confirmation of the experiments of Franck and Hertz.

After the cylinder and filament had been properly cleaned up, results were obtained of which the curves in fig. 3 serve as specimens. With the apparatus completely evacuated,

Fig. 3.

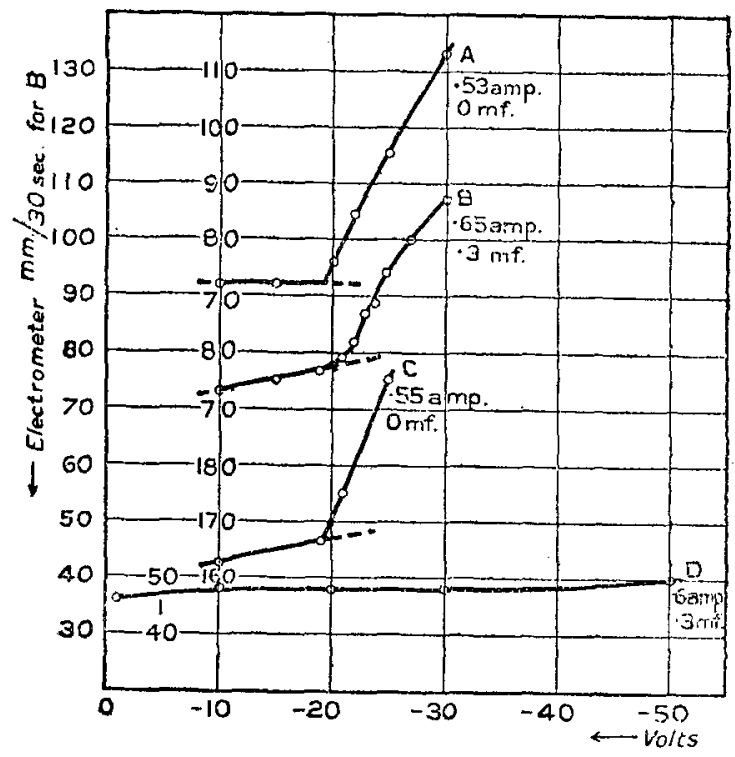

washed out repeatedly with air, and then reduced to a liquidair vacuum, the current-voltage curve has the form shown in D. Saturation, which is nearly reached at -1 volt, is complete at -10 volts, after which the current remains practically constant. In $B$ the apparatus contained pure helium at a pressure of about $.85 \mathrm{~mm}$. Liquid air had been on the U tube for six hours, but the green line of mereury was still faintly visible in the spectrum. The electron currents here were of such magnitude, due to the relatively high temperature of the filament, that $\cdot 3$ microfarad was necessary across the quadrants of the electrometer. Under these conditions complete saturation was not attained. The curve, nevertheless, shows clearly that impact ionization sets in when the potential applied is 19.5 volts. Curves of which $A$ is a specimen were obtained after liquid air had been on the $U$ tube for 24 liours or more, and the spectrum contained 


\section{Dr. C. B. Bazzoni : Experimental Determination}

no trace of mercury or of any other impurity. The electron currents were also reduced by lowering the temperature of the filament until no capacity was necessary across the electrometer. Here saturation is complete at 10 volts, and ionization is seen to set in at 19.5 volts. Curve $C$ was taken after the liquid air had evaporated, and a trace of mercury was present. The mercury, which presumably ionizes under 4.9 volt impacts, makes it impossible to get complete saturation, but, nevertheless, the amount present is too small to obscure the setting in of the helium ionization, which is again seen to start at 19.5 volts.

In order to deduce the correct ionization potential from these data, it is necessary to consider two disturbing factorsfirst, the drop of potential along the filament and, second, the initial velocity with which the electrons leave the wire. The first factor might be important because the filament is cool at the ends, so that no electrons are liberated exactly where the voltmeter is connected. The total drop along the filament with the currents used was found to be $\cdot 75$ volt. Allowing one-tenth of the length of the filament at each end as the part where the cooling effect would be appreciablewhich, judging from a visual examination, was an ample allowance-the corresponding correction to be applied to the voltmeter readings is seen to be 0.1 volt. The distribution of velocities amongst the emitted electrons was studied by applying positive potentials to the filament, and it was found that the maximum velocity of emission at the temperatures used was one volt. It is seen, therefore, that when the voltmeter read 19.5 volts there were some electrons coming from the negative end of the filament with velocities corresponding to 20.4 volts. The results are consequently in general accordance with those of Franck and Hertz. If the true value is different from $20^{\circ} 4$ volts, it is more likely to be somewhat below this value than above it, since very few of the electrons have initial velocities approaching one volt. The experimental arrangement, however, with a large anode completely surrounding the filament, and with the greater part of the drop of potential close to the filament, is a sensitive one, and ought to detect the ionization even when the number of electrons impinging with the necessary energy is small.

The phenomena of ionization in gases like helium and mercury vapour, involving, as they apparently do, elastic collisions below a certain velocity, and totally inelastic ones above that velocity, are worthy of close study. The hypothesis of a critical velocity for a totally inelastic impact was 
introduced by Franck and Hertz to explain the results of certain of their experiments, and seems in general to be in accordance with the facts. The ordinary interpretation of this hypothesis leads to the statement that an electron cannot exist in mercury vapour with a velocity above 5 volts, or in helium with a velocity above about 20 volts, provided that the dimensions of the receptacle are sufficiently large compared with the mean free path of an electron at the given pressure. A check on this hypothesis can be obtained from a consideration of the successive current maxima at multiple values of the critical voltage. With an apparatus like that used by Franck and Hertz, in which the positive ions are dealt with, no conclusion can, however, be drawn from the successive values of the current on account of the retarding effect of the accumulated positive ions. In mercury the successive maxima appear to be, as a matter of fact, nearly equal one to the other. In studying this point with the apparatus used in the present investigation, the pressure and the filament temperature were necessarily much reduced in order to get that approach to saturation in each successive current which was needed to allow the current increase at the succeeding critical value to be apparent. The pressure worked with $(\cdot 2 \mathrm{~mm}$.) was unfortunately one for which the mean free path of the electrons is not sufficiently small, compared with the radius of the receiving cylinder, to render it safe to draw definite conclusions from the observations. The results obtained are illnstrated by the curves of fig. 4 , in which the electron current arriving at the copper cylinder is plotted against the accelerating voltage. $\mathbf{A}$ is a preliminary curve in which particular attention was paid to the points around 40 volts. In B the points are carried to 85 volts. It will be observed that a definite increase takes place at multiple values of the ionization potential, but that the successive currents are not related to the original current in a 1.2.4.8 ratio, as would be the case if every electron collided inelastically with an atom and there were no disturbances due to positive layers. The only effects which can be produced by the positive ions in this experiment are, first, their very marked effect on the distribution of potential-a matter not yet sufficiently investigated - and, second, their action in removing a certain number of free electrons by recombination. The current ratios taken from curve B are, roughly, $1 \cdot 1 \cdot 25 \cdot 1 \cdot 76 \cdot 2 \cdot 18$. On the assumption that three tenths of the electrons undergo collisions at the specified ranges these numbers would be $1 \cdot 1 \cdot 30 \cdot 1 \cdot 69$ and $2 \cdot 20$. This 
proportion is about what would be expected from the dimensions of the apparatus and from the pressure of the heliuin used. It syems doubtful whether electron collisions with helium under the ionizing potential are perfectly elastic, but it is difficult to draw definite conclusions about this question from the present experiments.

Fig. 4.

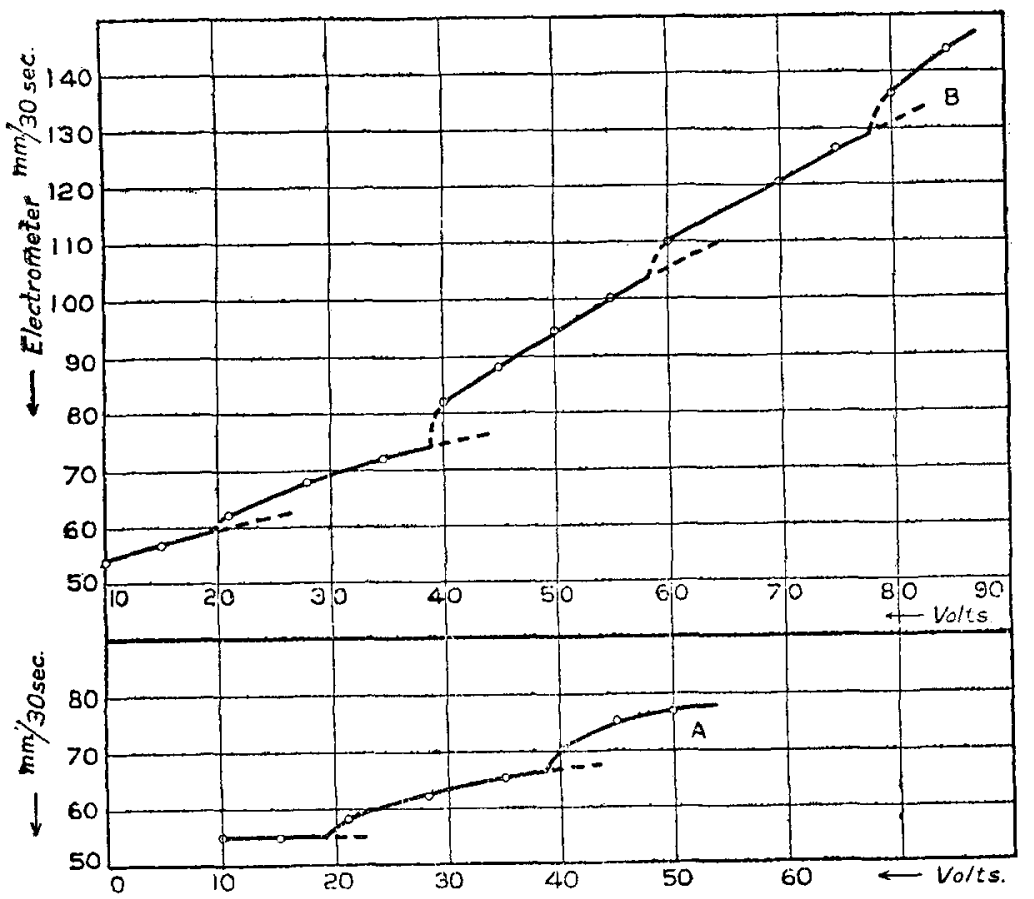

The curves of fig. 4 also indicate strongly that the true ionization potential of helium is not more than 20 volts, rather than 20.5 or 21 volts. This follows from the fact that the jump in ionization is seen already to have taken place at 40,60 , and 80 volts, at which potentials the correction to allow for initial velocities is relatively unimportant.

In conclusion, it is to be observed that the results obtained in these experiments cannot be ascribed either to the ionization of impurities-for there were no impurities present in the critical experiments-or to the liberation of electrons from the metal parts of the apparatus, for this would have registered a positive charge on the electrometer. It would 
seem, therefore, definitely established that ionization takes place in helium under an applied potential of about 20 volts. This fact can be reconciled with Bohr's theory only by making cortain new assumptions as to the mechanism of impact ionization in helium. These new assumptions have not yet been subjected to experimental investigation.

I wish to express my obligation to Professor $0 . W$. Richardson for valuable advice given throughout the course of these experiments. The quartz tube was constructed by Mr. Reynolds of the Silica Syndicate.

Wheatstone Laboratory,

King's College.

August 20, 1916.

LXIII. The Compression of the Earth's Crust in Cooling. $B y$ Harold Jefrereys, B.A., M.Sc., Fellow of St. John's College, Cambridge*.

\section{Introduction.}

$\mathrm{T}$ is generally agreed among geologists that the principal 1 cause of the elevation of continents and mountains is that the crust of the earth must be in a state of horizontal compression, under which it frequently gives way, the strata being then folded into a shorter length in the neighbourhood of the point where the weakness has been shown. Such a compression appears to be the only mechanism that has been suggested that is qualitatively capable of producing the observed results. The cause of the compression itself is, however, very uncertain. The contraction hypothesis is the most satisfactory of those that have been offered, but grave doubts have frequently been expressed about its quantitative adequacy. According to this hypothesis, the earth was originaliy at a very bigh temperature, and different parts have since cooled by different amounts, changing in volume in consequence. A state of strain is thus set up in the crust. The mathematical aspect of the theory is due originally to Dr. C. Davison $†$ and Sir G. H. Darwin $\ddagger$; they showed that as the inner layers after a certain time are cooling more rapidly than the outer ones, and therefore contracting more rapidly, the outer layers will have to undergo compression

* Communicated by the Author.

+ Phil. Trans. 178 A, pp. 231-242 (1887).

\pm Phil. Truns. 178 A, pp. 242-249 (1887); or Sci. Papers, vol. ir. p. 354. 